\title{
Resources Innovation Center an der Montanuniversität Leoben - Ressourcenforschung im Kontext der globalen Entwicklung
}

\author{
Peter Moser und Susanne Feiel
}

Resources Innovation Center Leoben, Montanuniversität Leoben, Leoben, Österreich

Eingegangen 28. Januar 2019; angenommen 4. Februar 2019; online publiziert 12. Februar 2019

Zusammenfassung: Das Resources Innovation Center Leoben an der Montanuniversität Leoben bündelt die internationalen Beteiligungen der Universität im Bereich der nachhaltigen Rohstoffforschung und Ausbildung. Von überragender Bedeutung für das Resources Innovation Center Leoben ist die Arbeit im EIT RawMaterials, einer sogenannten Knowledge \& Innovation Community des European Institute for Innovation and Technology, einem paneuropäischen Netzwerk aus 125 Partnern im Rohstoffbereich. Besonders in den Bereichen Bildung sowie Sustainable Exploration \& Mining, Technological Innovation und Recycling ist die Montanuniversität hier tätig. Zusätzlich bringt sie sich bei der strategischen Weiterentwicklung der Community ein. Ebenfalls von großer Bedeutung ist die Mitgliedschaft in der EIT Climate-KIC, einer weiteren Knowledge \& Innovation Community. Im EIT Climate-KIC werden die Themen Urban Transitions und Sustainable Production Systems vorangetrieben, um die Auswirkungen des Klimawandels zu begrenzen und eine klimaresiliente Gesellschaft zu fördern. Ein zusätzlicher Bereich im Aktivitätsportfolio des Resources Innovation Center Leoben sind die Sustainable Development Goals der Vereinten Nationen, zu denen durch vielerlei Initiativen beigetragen wird. Eine Schlüsselaktivität in diesem Bereich ist die Implementierung der Sustainable Development Goals im österreichischen Hochschulsektor durch ein gemeinsames Netzwerkprojekt aller österreichischen Universitäten. Eines haben alle Arbeiten im Resources Innovation Center Leoben gemein: Sie sind thematisch miteinander verknüpft und ergänzen sich. Die Resultate, die es zu erzielen gilt, zielen allesamt auf nachhaltige Innovation im Ressourcenbereich für eine bessere Zukunft ab.

\footnotetext{
Univ.-Prof. Dipl.-Ing. Dr. mont. P. Moser ( $\square)$ Resources Innovation Center Leoben,

Montanuniversität Leoben,

Franz Josef-Str. 18,

8700 Leoben, Österreich

peter.moser@unileoben.ac.at
}

Schlüsselwörter: Montanuniversität Leoben, EIT RawMaterials, EIT Climate KIC, Knowledge \& Innovation Community, Regional Center Leoben, Globalisierung, Nachhaltigkeit, Internationalisierung, Innovation, Rohstoffe, Resources Innovation Center Leoben

Resources Innovation Center at Montanuniversitaet Leoben-Resources Research in the Context of Global Development

Abstract: The Resources Innovation Center Leoben at Montanuniversitaet Leoben is home to the international partnerships of the University in the areas of sustainable resources research and education. Of prime importance is the Resources Innovation Center's participation in the EIT RawMaterials, a pan-European network for raw materials, a so-called Knowledge \& Innovation Community of the European Institute for Innovation and Technology, recently comprising 125 partners. In particular, the Resources Innovation Center Leoben works on projects in the areas of education, sustainable exploration \& mining, technological innovation and recycling. It is also an active partner in terms of the strategic development of the community. Another activity of great importance is the participation in the EIT Climate-KIC, which is also a Knowledge \& Innovation Community. Here we focus upon the areas of Urban Transition and Sustainable Production Systems to decrease greenhouse gas emissions and foster a climate resilient society. Another pillar at the Resources Innovation Center Leoben is sustainable development, in which we are committed to achieving the United Nations Sustainable Development Goals through various initiatives. One of the key activities in this respect is the contribution to the Austrian Higher Education sector through a joint network project of the majority of all Austrian universities. All activities of the Resources Innovation Center are thematically connected and synergetic. They are aiming at sustainable innovation in the resources sector for a better future. 
Keywords: Montanuniversitaet Leoben, EIT RawMaterials, EIT Climate KIC, Knowledge \& Innovation Community, Regional Center Leoben, Globalised world society, Sustainable Development Goals, Innovation, Raw Materials, Resources Innovation Center Leoben

\section{Globale Ressourcendiskussion und Entwicklungen an der Montanuniversität Leoben}

Die wichtigsten gesellschaftlichen Herausforderungen der Zukunft, deren Bearbeitung für Universitäten gleichsam eine Pflicht ist, sind heute ausnahmslos globale Problemstellungen. Diese finden sich in den von den Vereinten Nationen (UN) im September 2015 verabschiedeten 17 globalen Entwicklungszielen (https://www.un. org/sustainabledevelopment/sustainable-developmentgoals/) wieder.

Sie haben die weltweite Erhöhung des Wohlstandes und insgesamt das Verbessern der Lebenssituation zum Ziel. Allen Menschen soll Zugang zu Bildung, einer guten medizinischen Versorgung, einer adäquaten Infrastruktur und einer intakten Umwelt ermöglicht werden. Mit den globalen Entwicklungszielen verbunden sind eine massive Ausweitung der industriellen Produktion und ein steigender Rohstoffbedarf, der sich nach vorsichtigen Schätzungen bis zum Jahre 2050 von zurzeit rund $40 \mathrm{Mrd}$. t p. a. auf rund $80 \mathrm{Mrd}$. $\mathrm{t}$ p. a. verdoppeln wird. $80 \%$ der global benötigten Rohstoffe sind mineralische Rohstoffe, die dem Bergbau entstammen. Diese umfassen Energierohstoffe, Baurohstoffe, Industrieminerale und Metalle. Nur rund $20 \%$ sind biogene Rohstoffe aus der Land- und Forstwirtschaft [1]. Diese Ausweitung der industriellen Produktion verlangt nach neuen Produktions- und Fertigungstechnologien, um die zunehmende Weltbevölkerung innerhalb der Umweltgrenzen des Planeten Erde nachhaltig versorgen zu können.

\section{Resources Innovation Center Leoben}

Zur Bündelung der Beiträge für eine nachhaltige Versorgung der Gesellschaft mit leistbaren Rohstoffen wurde 2015 an der Montanuniversität Leoben das RIC-Resources Innovation Center Leoben (https://www.ric-leoben.at/) gegründet, welches die Beteiligungen der Montanuniversität an internationalen Rohstoff-, Klima- und Nachhaltigkeitsinitiativen bündelt. Dies betrifft $u$. a.:

- Knowledge \& Innovation Community EIT RawMaterials

- Knowledge \& Innovation Community EIT Climate-KIC

- Forschungs- und Ausbildungsprojekte im Rohstoffbereich im Rahmen von Horizon 2020 und Horizon Europe

- Climate Change Centre Austria

- Sustainable Development Goals (SDGs)

- European Innovation Partnership on Raw Materials

- Universitäten und Nachhaltige Entwicklungsziele (UniNEtZ)

- Allianz Nachhaltige Universitäten
- World Forum of Universities of Resources on Sustainability (WFURS)

- UN Sustainable Development Solutions Network (SDSN)

Am Anfang der RIC Aktivitäten stand die Beteiligung an der Knowledge \& Innovation Community (KIC) EIT RawMaterials, einem paneuropäischen Netzwerk des „European Institute for Innovation and Technology" im Rohstoffbereich. Eine KIC besteht aus einem Konsortium aus Industrie, Forschungsinstitutionen und Universitäten, welches gesellschaftliche Herausforderungen in Form von Innovations- und Bildungsprojekten bearbeitet. Ebenfalls von Bedeutung ist die Mitwirkung an der Knowledge \& Innovation Community EIT Climate-KIC. Im EIT Climate-KIC werden die Themen Urban Transitions und Sustainable Production Systems vorangetrieben, um die Auswirkungen des Klimawandels zu begrenzen und eine klimaresiliente Gesellschaft zu fördern. Ein zusätzlicher Bereich, der im Aktivitätenportfolio des RIC Leoben aufscheint, ist die Befassung mit den Sustainable Development Goals (SDGs), zu denen sich die internationale Staatengemeinschaft 2015 verpflichtet hat. Eine Schlüsselaktivität in diesem Bereich ist die Implementierung der SDGs im österreichischen Hochschulsektor durch ein gemeinsames Netzwerkprojekt aller österreichischen Universitäten (UniNEtZ).

Eines verbindet alle RIC Aktivitäten: Sie sind thematisch miteinander verknüpft und ergänzen sich, indem die Resultate, die es zu erzielen gilt, allesamt auf nachhaltige Innovation im Ressourcenbereich für eine bessere Zukunft abzielen.

Die Aktivitäten des Resources Innovation Center Leoben werden sowohl aus Mitteln der Montanuniversität als auch durch Sondermittel des Bundesministeriums für Bildung, Wissenschaft und Forschung (BMBWF) und des Bundesministeriums für Digitalisierung und Wirtschaftsstandort (BMDW) finanziert. Eine weitere wesentliche Finanzierungsquelle stellt die umfangreiche Beteiligung an Projekten auf Europäischer Ebene dar (H2020, EIT Raw Materials, EIT Climate-KIC).

\section{EIT RawMaterials}

Von all den wichtigen internationalen Partnerschaften im Rohstoffbereich muss dem EIT RawMaterials besondere Aufmerksamkeit geschenkt werden, da die Montanuniversität nicht nur Gründungsmitglied dieser Knowledge and Innovation Community ist, sondern auch sehr stark gestalterisch in dieser Gemeinschaft mitwirkt. Kurz zusammengefasst, handelt es sich beim EIT RawMaterials um das größte Netzwerk weltweit, in dessen Fokus Rohstoffe stehen. Es wurde vom EIT - dem European Institute of Innovation and Technology - als Werkzeug ins Leben gerufen, sich den gesellschaftlichen Herausforderungen Europas zu stellen, die sich durch Themen wie Klima, Informations- und Kommunikationstechnologien sowie Rohstoffe definieren.

Das Aktivitätsportfolio des EIT RawMaterials erstreckt sich über die gesamte Wertschöpfungskette, da der Begriff "Raw Materials" auf EU Ebene weit gefasst und der Rohstoff selbst in seiner gesamten Lebensdauer bis hin zur Wie- 
dereinführung in den Kreislauf (Recycling) betrachtet wird. Diese Sichtweise deckt sich direkt mit den Kernkompetenzen der Montanuniversität und ihrer Fokussierung auf den Wertschöpfungskreislauf [2]. Schwerpunktmäßig gruppieren sich die Forschungs- und Bildungsprojekte rund um folgende Felder:

- Exploration \& Ressourcen-Bewertung

- Bergbau im herausfordernden Umfeld sowohl hinsichtlich technologischer als auch gesellschaftlicher Parameter

- Ressourcen-Effizienz in der Aufbereitung und Metallurgie

- Recycling \& Stoffflussoptimierung

- Substitution von kritischen Rohstoffen \& toxischen Materialien in Produkten

- Design für Produkte \& Dienstleistungen für die Kreislaufwirtschaft

Als jüngster Schwerpunkt wurde die Befassung mit Rohstoffen und E-Mobilität gesetzt.

Innerhalb des EIT RawMaterials Konsortiums hat die Montanuniversität eine Rohstoffstrategie für Ost- und Südosteuropa (ESEE Region - East and South East Europe) entwickelt. Die ESEE Region verfügt über Vorkommen wichtiger kritischer Rohstoffe. Potenziale werden sowohl bei der Neuerschließung von Lagerstätten als auch bei der Aufbereitung sekundärer Rohstoffe, etwa alter Halden sowie Schlacken gesehen. Eine Realisierung der Potenziale wird die ökonomische Entwicklung der Region voranbringen und damit einen Beitrag zu deren sozialer sowie auch politischer Weiterentwicklung leisten [3]. Zwischen 2015 und 2018 wurden von der Montanuniversität in Ländern Ost- und Südosteuropas bisher insgesamt neun „ESEE Dialogue Conferences" abgehalten, mit dem Ziel, das Thema Rohstoffe in den politischen und wirtschaftlichen Fokus von Forschung und Ausbildung zu stellen. Als Ergebnis wurden nicht nur umfangreiche Projektaktivitäten mit Beteiligung der Montanuniversität und vieler EIT RawMaterials-Partner aus ganz Europa gestartet, sondern auch die Einrichtung von "Rohstoff Hubs" in Laibach und Zagreb (Regional Hub Adria) und Athen (Regional Hub Greece) realisiert. Im Hinblick auf die Weiterentwicklung der Ausbildung im Rohstoffbereich wurde eine „Raw Materials ESEE Education Strategy" entwickelt, die nun eine Leitlinie für die Entwicklung des Bildungssektors im Rohstoffbereich darstellt.

In der EIT RawMaterials Community sind momentan insgesamt rund 125 Partner mit Rohstoffinteressen beteiligt. Diese bewegen ein unmittelbares Forschungsvolumen von rund $€ 100$ Mio. mit zusätzlich etwa 3-fachen komplementären Aktivitäten der Mitglieder. Nach Österreich flossen über die EIT RawMaterials-Beteiligung bisher Fördergelder in der Höhe von rund $€ 12$ Mio.

Für die Montanuniversität zeigen sich erhebliche Vorteile aus der Teilnahme an dieser Forschungscommunity insbesondere bei der Bildung großer Projektkonsortien und bei der Erarbeitung von komplexen Projektideen. Aber auch das Netzwerk an sich stellt schon einen erheblichen Mehrwert dar. Durch die starke synergetische Vernetzung der Partner können schlussendlich gemeinsam auch andere Programme zum Forschungsinnovationsantrieb genutzt werden. Seit der Gründung des EIT RawMaterials im Jahre 2015 und durch Nutzung von Partnersynergien wurde beispielsweise eine erhebliche Zahl an Horizon 2020 Forschungsprojekten und Projekten des Europäischen Fonds für regionale Entwicklung im Rohstoffbereich mit Beteiligung der Montanuniversität beantragt und gewonnen. Diese sind:

- MinGuide: Mineral Policy Guidance for Europe (https:// www.min-guide.eu/)

- Slim: Sustainable Low Impact Mining solution for exploitation of small mineral deposits based on advanced rock blasting and environmental technologies (https://www. slim-project.eu/)

- Iterams: Integrated Mineral Technologies for More Sustainable Raw Material Supply (http://www.iterams.eu/)

- Minland: Mineral resources in sustainable land-use planning (http://minland.eu/)

- Mireu: Mining and Metallurgy Regions of Europe (https:// mireu.eu)

- Intermin: International Network of Raw Materials Trainings Centres (http://interminproject.org/)

- Remix: Smart and Green Mining Regions of EU (Interreg Projekt) (https://www.interregeurope.eu/remix/)

- Robominer: Resilient Bio-inspired Modular Robotic Miners

Auch die Förderung innerhalb des EIT RawMaterials ist respektabel: In den letzten drei Jahren wurde die Montanuniversität in insgesamt 37 Forschungs- und Ausbildungsprojekten - KAVA (KIC-Added-Value-Activities) Projekten aktiv. Ein sehr anspruchsvolles technisches Projekt ist zum Beispiel das KAVA Projekt Rock Vader, das gemeinsam mit der Firma Sandvik in Zeltweg, dem Wolframbergbau Mittersill und weiteren europäischen Partnern verwirklicht wird. Es geht dabei um eine neue Hartgesteinsvortriebsmaschine mit einer innovativen Technik an den Schneidköpfen. Diese neue Technologie eröffnet in der Zukunft die Möglichkeit, im untertägigen Erzbergbau deutlich höhere Auffahrungsleistungen zu erzielen. Ein anderes technisches Projekt, das sich dem Recycling widmet, ist das KAVA Projekt WEE REC. Dieses Projekt hat zum Ziel, eine neue Technologie für das Recycling von minderwertigem Elektronikschrott zur Marktreife zu führen. Dies hat nicht nur einen positiven Effekt auf die Umwelt durch einen Kreislaufeffekt, sondern sorgt ebenfalls dafür, dass minderwertiger Elektronikschrott als Sekundärrohstoff in Europa aufgearbeitet werden kann und nicht exportiert werden muss.

Ein KAVA Ausbildungsprojekt, das darauf abzielt, die Rohstoffausbildung in Europa zukünftig auf die Basis digitalisierter Lehrbücher zu stellen und damit neue europa- und weltweite Standards zu schaffen, nennt sich „MiReBooks Mixed Reality Handbooks for Mining Education“. Unter der Führung der Montanuniversität Leoben arbeiten europaweit eine Reihe von Bergbauuniversitäten, Forschungsinstitutionen, Bergbauausrüstern und Bergbaufirmen daran, Lehrunterlagen und Bergbauhandbücher so aufzubereiten, dass mit Hilfe moderner elektronischer Technologie auf Ba- 
sis von Virtual und Augmented Reality eine 3-D Bergbauumgebung in die Hörsäle transportiert wird und für eine realitätsnahe Ausbildung eingesetzt werden kann.

\section{EIT Climate-KIC}

Die EU-Innovationsschmiede EIT Climate-KIC eröffnete am 1. Juni 2017 eine neue Geschäftsstelle in Österreich. Das RIC der Montanuniversität war hier mit weiteren drei österreichischen Partnern von Beginn an Bord: WWF Österreich, Brainbows und das Austrian Institute of Technology. Über 300 exzellente Partner aus Wirtschaft, Wissenschaft und öffentlicher Hand bilden das EIT Climate-KIC, Europas größtes öffentlich-privates Netzwerk für Innovationen gegen den Klimawandel. EIT Climate-KIC ist in 25 europäischen Ländern aktiv und verknüpft Innovationsförderung, ein europäisches Accelerator-Programm für Start-ups sowie hochwertige Bildungsangebote, um systematisch Wissen in wettbewerbsfähige Produkte und Dienstleistungen zu wandeln.

Die Montanuniversität bringt sich in dieses pan-europäische Forschungs- und Bildungsnetzwerk schwerpunktmäßig mit Kompetenzen in klimarelevanten Bereichen wie Wasserstoffmetallurgie, Hochtemperaturelektrolyse, Einsatz Erneuerbarer Energien (spezielle Biomasse) in der Nicht-Eisen-Metallurgie, CCU - Carbon Capture \& Utilisation (von $\mathrm{CO}_{2}$ zu Baustoffen, Gas to Power etc.), Geothermie, Energieeffizienz im industriellen Maßstab sowie Kreislaufwirtschaft ein. Mit neuen Partnern werden auch in diesen Themenfeldern interessante Innovationen angestoßen. Bereits im ersten EIT Climate-KIC Beteiligungsjahr erhielt das RIC zusammen mit vier internationalen Partnern den Zuschlag für das Flagship-Projekt "eCircular" (https://ecircular.climate-kic.org/), das sich auf die Kreislaufwirtschaft im Kunststoffbereich fokussiert. Ziel des Projektes ist die Vermeidung von Kunststoffabfällen durch die konsequente Anwendung digitaler Technologien, das Umsetzen von Eco-Design-Ansätzen sowie die Entwicklung von Industriestandards. Die Mitwirkung an diesem Flagship-Projekt ermöglicht es dem RIC, diesen Themenbereich strategisch mitzugestalten und die Arbeit der Montanuniversität Leoben international sichtbar zu machen. Es sind hierfür auch schon einige interessante Projekte aus dem Bereich Kunststofftechnik in Vorbereitung. So startet demnächst ein Vorprojekt zur Entwicklung des europäischen Marktes für Sekundärkunststoff, an dem neben der Montanuniversität Leoben auch die Universität Lund und die Firma E.ON beteiligt sind.

Auch im EIT Climate-KIC spielen Aktivitäten im Bereich der universitären Bildung eine wichtige Rolle. Hier war im Jahr 2018 die vom EIT Climate-KIC zusammen mit zahlreichen Partnern organisierte und durchgeführte Internationale Summer School "The Journey" (https://journey.climatekic.org/) erstmals in Österreich zu Gast. Über 400 Studierende haben dabei während einer fünfwöchigen Reise durch Europa klimafreundliche und nachhaltige Geschäftsideen entwickelt und diese an der Montanuniversität Leoben einer internationalen Jury vorgestellt.

\section{Climate Change Centre Austria}

Das Climate Change Centre Austria (CCCA) ist ein von Forschungsinstitutionen Österreichs getragenes Forschungsnetzwerk, das selbst keine Forschung durchührt, sondern als Koordinationseinrichtung das Erreichen der Forschungsziele mit dem Fokus auf die Klimaforschung vorantreibt (https://ccca.ac.at/de/home/). Die Montanuniversität Leoben ist dem CCCA 2018 beigetreten und engagiert sich seither mit CCCA-Partnern beim Ausloten und Entwickeln von Projekten im Zusammenhang mit ressourceneffizienter Fertigung und der Dekarbonisierung von Industrieprozessen sowie der Identifizierung von Risiken für die Industrie im Transformationsprozess zur $\mathrm{CO}_{2}$-Neutralität.

\section{Sustainable Development Goals (SDGs)- Globale Entwicklungsziele und Ressour- cen}

Ein weiterer Bereich, der im Aktivitätsportfolio des RIC Leoben seit 2017 aufscheint, sind die Sustainable Development Goals, welche die von den Vereinten Nationen im September 2015 verabschiedeten 17 globalen Entwicklungsziele beinhalten.

Eine Schlüsselaktivität in diesem Bereich ist die Erfassung und Ausarbeitung wissenschaftlicher Beiträge als Optionen, die gesetzt werden müssten, um die SDGs in Österreich umzusetzen. Die Allianz Nachhaltige Universitäten in Österreich, der die Montanuniversität seit 2018 angehört, hat dazu die Initiative ergriffen, die Umsetzung der SDGs in Österreich voranzutreiben. Unter dem Namen UniNEtZ - Universitäten und Nachhaltige Entwicklungsziele - wird ein Optionenpapier zur Umsetzung der SDGs in Österreich ausgearbeitet. Zusätzlich wird angestrebt, nachhaltiger Entwicklung an den Universitäten in Lehre und Forschung einen Platz zu sichern.

Das RIC befasst sich im Rahmen dieser Initiative schwerpunktmäßig mit den Entwicklungszielen SDG 7: Leistbare und saubere Energie, SDG 12: Nachhaltige Produktion und Konsum, SDG 4: Hochwertige Bildung und SDG 13: Maßnahmen zum Klimaschutz.

\section{European Innovation Partnership on Raw Materials - EIP Raw Materials}

Seit bereits mehr als 5 Jahren wirkt die Montanuniversität Leoben an der „European Innovation Partnership on Raw Materials" der Europäischen Generaldirektion für Binnenmarkt, Industrie, Unternehmertum und KMU mit. Sowohl in der "High Level Steering Gruppe" als auch in der "Sherpa Gruppe" sind die beiden Vertreter der Montanuniversität Leoben (Peter Moser, Susanne Feiel) die einzigen aus dem universitären Bereich in Europa und vertreten daher in der EIP Raw Materials die Interessen des Forschungsund Bildungssektors im Rohstoffbereich in ganz Europa. Schwerpunkte der Arbeit im Rahmen der EIP Raw Materials waren zunächst die Ausarbeitung eines Strategischen Konzepts für den Rohstoffsektor in Europa (Strategic Imple- 
mentation for the European Innovation Partnership on Raw Materials - Part 1 and Part 2) [4, 5] und dessen Implementierung. Mittlerweile umfassen die Arbeiten auch die Weiterentwicklung der strategischen Konzepte zur Einarbeitung in das zukünftige F\&E Programm Horizon Europe.

\section{Quo vadis - ein kurzer Ausblick}

Die Jahre 2015 bis 2018 waren im Resources Innovation Center an der Montanuniversität Leoben vom Aufbau neuer Strukturen bei gleichzeitigem Hochfahren entsprechender Forschungs-, Entwicklungs- und Ausbildungsaktivitäten geprägt. Während die Beteiligung am EIT RawMaterials organisatorisch und inhaltlich schon weitgehend gefestigt ist, müssen die Möglichkeiten im Rahmen der EIT ClimateKIC noch stärker ausgelotet und strategisch an die Montanuniversität geholt werden. Die verstärkte Integration österreichischer Unternehmen und die Forcierung nachhaltiger "Start-Up“ Aktivitäten, insbesondere im Rohstoff- und Klimabereich, stellen wesentliche Herausforderungen für die zukünftigen RIC-Aktivitäten dar. In fachlicher Hinsicht ist eine Fokussierung auf folgende Themen vorgesehen:

- Integrierte Nutzung von Rohstoffvorkommen in Europa und deren generationenübergreifende nachhaltige Entwicklung

- Ressourceneffizienz und wirtschaftliche Nutzung von bisher nicht genutzten Nebenprodukten und Abfällen

- Stoffflussanalysen und nachhaltiges Stoffflussdesign im Hinblick auf die Vision Circular Economy

- Integrierte Digitalisierung von Betrieben der Rohstoffgewinnung - von der Lagerstätte bis hin zum operativen Betrieb

- Digitalisierung der Ausbildung im ingenieurwissenschaftlichen Bereich unter Nutzung von AR\& VR Technologien
Im Bereich der Sustainable Development Goals liegt der Schwerpunkt der Arbeiten auf dem Entwickeln von operativen Plänen zur Umsetzung der UniNEtZ-Arbeiten und insbesondere auch auf dem Integrieren dieses Projektes in die Lehr- und Forschungsaktivitäten an der Montanuniversität.

Funding. Open access funding provided by Montanuniversität Leoben.

Open Access Dieser Artikel wird unter der Creative Commons Namensnennung 4.0 International Lizenz (http://creativecommons.org/licenses/ by/4.0/deed.de) veröffentlicht, welche die Nutzung, Vervielfältigung, Bearbeitung, Verbreitung und Wiedergabe in jeglichem Medium und Format erlaubt, sofern Sie den/die ursprünglichen Autor(en) und die Quelle ordnungsgemäß nennen, einen Link zur Creative Commons Lizenz beifügen und angeben, ob Änderungen vorgenommen wurden.

Hinweis des Verlags. Der Verlag bleibt in Hinblick auf geografische Zuordnungen und Gebietsbezeichnungen in veröffentlichten Karten und Institutsadressen neutral.

\section{Literatur}

1. Moser, P.; Tost, M.; Feiel S.: Today's global overall resources consumption and the development since 1990, Vortrag. Alpbach Technology Symposium Breakout Session 12: The Renewability of Societal Resources (24.08.2018)

2. Montanuniversität Leoben: Entwicklungsplan der Montanuniversität Leoben 2017. Stand 6. Dezember 2017, Mitteilungsblatt der Montanuniversität Leoben, 033 - 2017/2018. Leoben: Dezember 2017

3. Maier, A.; Moser, P.: Rohstoffinitiativen auf europäischer Ebene, BHM Berg- und Hüttenmännische Monatshefte 160 (2015), H. 4, S. 175-182

4. European Commission, DG Enterprise and Industry: Strategic Implementation for the European Innovation Partnership on Raw Materials - Part 1, Final version 18/09/2013

5. European Commission, DG Enterprise and Industry: Strategic Implementation for the European Innovation Partnership on Raw Materials - Priority areas, Action areas and Actions. Part 2, Final version 18/09/2013 